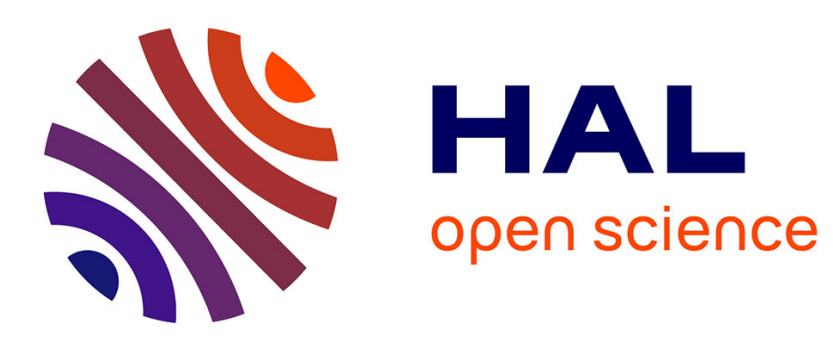

\title{
The Healthy Core Metabolism: A New Paradigm for Primary Preventive Nutrition
}

\author{
Anthony Fardet, Edmond Rock
}

\section{To cite this version:}

Anthony Fardet, Edmond Rock. The Healthy Core Metabolism: A New Paradigm for Primary Preventive Nutrition. Journal of Nutrition, Health \& Aging, 2016, 20 (3), pp.239-247. 10.1007/s12603015-0560-6 . hal-01458429

\section{HAL Id: hal-01458429 \\ https://hal.science/hal-01458429}

Submitted on 6 Feb 2017

HAL is a multi-disciplinary open access archive for the deposit and dissemination of scientific research documents, whether they are published or not. The documents may come from teaching and research institutions in France or abroad, or from public or private research centers.
L'archive ouverte pluridisciplinaire HAL, est destinée au dépôt et à la diffusion de documents scientifiques de niveau recherche, publiés ou non, émanant des établissements d'enseignement et de recherche français ou étrangers, des laboratoires publics ou privés. 


\title{
THE HEALTHY CORE METABOLISM: A NEW PARADIGM FOR PRIMARY PREVENTIVE NUTRITION
}

\author{
A. FARDET, E. ROCK \\ 1INRA, Human Nutrition Department, JRU 1019, UNH, CRNH Auvergne, F-63000 Clermont-Ferrand \& Clermont Université, Université d'Auvergne, Unité de Nutrition Humaine, \\ BP 10448, F-63000 Clermont-Ferrand, France. Corresponding author: Dr. Edmond Rock, INRA, Human Nutrition Department, JRU 1019, UNH, CRNH Auvergne, F-63000 Clermont- \\ Ferrand \& Clermont Université, Université d'Auvergne, Unité de Nutrition Humaine, BP 10448, F-63000 Clermont-Ferrand, France, +33 (0)4 73624169 , fax +33 (0)4 73624638 , \\ E-mail: edmond.rock@clermont.inra.fr
}

\begin{abstract}
Research in preventive nutrition aims at elucidating mechanism by which our diet helps us to remain in good health through optimal physiological functions. However, despite decades of accumulated data in human nutrition and regular subsequent nutritional recommendations, obesity and type 2 diabetes epidemics continue to progress worldwide each year leading to a regular decrease of the Healthy Life Years, notably in Western countries. Such a paradox may be explained by the Nutrition Transition, the extreme application of the reductionist paradigm in nutrition research, the lack of nutritional education and a too strong focus on curative nutrition in at risk/ill subjects. In this position paper, we hypothesized that researchers should focus more on healthy subjects, from birth until maturity. Rather than exploring what differentiates healthy and at risk/ ill subjects, we propose to thoroughly study what characterizes a healthy state and its underlying metabolism. We define it as the Healthy Core Metabolism which remains stable whatever energy inputs (diets) and outputs (exercise), genetic background and external/internal stress, e.g., temporary illnesses. As a basis for Healthy Core Metabolism investigation, we observed that main physiological and ubiquitous functions of human organism, i.e., the neuro-vasculo-sarco-osteoporotic system, tend to follow a concave curve with common phases of growth, optimum, and decline. Finally, we hypothesized that true primary preventive nutrition should focus on the growth phase to reach the maximum capital of a given physiological function so that - whatever the further decline -, Healthy Life Years may approach or coincide with theoretical Life Expectancy.
\end{abstract}

Key words: Healthy core metabolism, preventive nutrition, metabolic optimum, healthy metabolic range.

\section{Introduction}

The World Health Organization has proposed to cluster prevention in 3 categories: 1) Primary prevention includes all actions intended to reduce the incidence of a disease in a population, thereby reducing the risk of occurrence of new cases. It uses measures of individual (personal hygiene, diet, physical activity and sport, vaccinations ...) and collective (drinking water, waste disposal, food safety, immunization, hygiene and habitat workplace) prevention. This traditional concept of prevention inevitably leads to a vast improvement in the quality of life and social institutions reform program; 2) Secondary prevention includes «all acts intended to reduce the prevalence of a disease in a population, thereby reducing the duration of disease progression». It takes into account the early detection and treatment of first symptoms; 3) Tertiary prevention includes all actions intended to reduce the prevalence of chronic disability or recurrence in a population, so as to minimize the functional consequences of disability from the disease; it extends the prevention to the field of rehabilitation by seeking to promote professional and social reintegration.

In theory, research in preventive nutrition aims at elucidating the mechanism by which our diet help us to remain in good health, through optimal physiological functions. Today, preventive nutrition also associates physical activities, and more generally gaining a sense of lifestyle choices and their effects upon one's health. The final objective is therefore

Received January 19, 2015

Accepted for publication March 3, 2015 to achieve optimal health and to live a long life in good health, idealistically to align the Healthy Life Years with Life Expectancy. Therefore, the target of this primary prevention is clearly the healthy large public. Preventive nutrition is then ensured by public health policies via different mode of communication such as food pyramids, nutritional education, national nutrition programs such as the French Programme National Nutrition Santé (PNNS, http://www.mangerbouger.fr/ pnns).

Yet, diet-related chronic diseases prevalence increases each year, notably for type 2 diabetes, obesity, cancers and cardiovascular diseases. It then appears that preventive nutrition did not succeed in stopping such epidemics despite several decades of research and the huge amount of nutritional data collected (1). Several reasons may be proposed for this situation: first, the well-known nutrition transition from traditional, unrefined, nutrient-dense and minimally-processed foods to ultra-processed and energy-dense foods and the parallel reduction of physical activities together with increased sedentarity (2), second, the application of the reductionist paradigm associating one nutrient with one physiological effects and reducing food health potential to only a few compounds and that have led to fractionation-recombination of foods and to consider preventive nutrition according to a pharmacological approach on the one hand and food bioactives similar to drugs on the other hand (1), and third the generally low level of nutritional education leaving most populations powerless in front of marketing pressure (e.g., advertisements 


\section{HEALTHY CORE METABOLISM AND PREVENTIVE NUTRITION}

and labeling, risky health claims, etc.) by agro-food industry and related stakeholders for ultra-processed and/or recombined foods with high monetary added value.

As a result, the Healthy Life Years progressively decrease each year leaving an always longer duration of life with disabilities, notably due to diet-related chronic diseases. The duration with disabilities is today above 20 years in France for both women (between 64, Healthy Life Years, and 85 years, Life Expectancy) and men, which is more and more humanly and economically unbearable (1). Yet, in 1980, Fries et al. introduced the Compression of Morbidity hypothesis, " positing that the age of onset of chronic illness may be postponed more than the age at death and squeezing most of the morbidity in life into a shorter period with less lifetime disability » (3). Preventive nutrition and healthy diets may therefore be expected to significantly extend the Healthy Life Years nearer to the age at death (4). Yet, until today, preventive nutrition has been mainly focusing on secondary and tertiary prevention that is preventing at risk people or curing ill subjects from developing chronic diseases. In addition, research in preventive nutrition has primarily investigated differences between at risk/ill and healthy subjects to unravel potential differences in physiological states (5). Paradoxically, the healthy state itself is not so well characterized and much remains to be done in this field of research. Yet, a thorough knowledge of the healthy state and of factors that allow an individual to remain in a healthy state is of utmost importance to set a solid primary preventive nutrition so that most people may live a less long unhealthy life.

The main objective of this position paper is to define new concepts and paradigms for studying the healthy state in humans; and thereafter to redefine preventive nutrition based on these new concepts. Finally, new research issues and perspectives based on this new preventive nutrition are proposed.

\section{How is defined a healthy sate? The current paradigms}

When we go for a medical assessment, we are all familiar with the healthy plasma levels of some current biomarkers such as triglycerides, cholesterol, glucose, iron, ALAT or ASAT, and of the minimal and maximal values not to exceed. Generally, most people think that to be healthy is the absence of (chronic) illnesses. But the WHO defines health as: "Health is a state of complete physical, mental and social well-being and not merely the absence of disease or infirmity" (6).

The objectives of medical assessments are also to detect as soon as possible biomarkers of upcoming diseases. When outside minimal and maximal values, the usual above-cited biomarkers already indicate a physiological dysfunction if not a disease. Today, thanks to metabolomics (7), one is able to define prognostic, early and diagnostic, and late biomarkers of diseases (8), which is an important breakthrough for secondary prevention. This evidently applies to diet- related chronic diseases like obesity (9), type 2 diabetes (10), cardiovascular diseases (11) and cancers (12). But to the best of our knowledge, the healthy state remains to be defined or characterized via metabolomics. Indeed, most human studies were interested by what differentiate ill versus healthy populations, not with what is common to them.

Today, it is clear that preventive nutrition is rarely applied very early in human life, but most of the time in the second half of life where the individuals are already at risk if not affected by degenerative diseases. The development of functional foods and supplements (i.e., nutraceuticals) for the latter population are more for curing an already unbalanced diet than for avoiding illness (1). And if nutraceuticals fail to restore a healthy state, this leaves place for pharmacological industry and medicine. It seems that a real primary nutrition-based prevention should be applied very early, at least during the first half of life, and idealistically starting by the mother before child conception. Yet, today, researchers in nutrition seem more focused on the health state of elderly than that of infants, young children, adolescent and young adults (i.e., before midlife); the elderly having a higher purchasing power than the youngest!

Therefore, from a scientific perspective there is an understudied issue: to define a clear and precise definition of the healthy state and whole body metabolic characteristics before appearance of prognostic biomarkers, and the way this healthy metabolic profile - which we define for the first time as the Healthy Core Metabolism (HCM) - evolve in time depending on energetic inputs (diet) and energy output (physical activities). Collecting and interpreting data about the HCM are probably the first step for efficient nutritional recommendation within the framework of primary prevention using diets instead of foods or bioactives. As Rezzi et al. wrote: "A major charter for modern nutrition is to provide a molecular basis for health outcome resulting from different food choices and how this could be designed to maintain individual health free of disease" (page 251) (13).

\section{The frontier between health and illness}

\section{The neuro-vasculo-sarco-osteoporotic system}

Today, the research in preventive nutrition intends to unravel food factors that may help to preserve a good health and/ or to re-equilibrate an unbalanced diet, most of the time in old subjects, people at risk or ill patients. With the advent of metabolomics, we can attempt to define more precisely the line - if it really exists! - between a healthy and unhealthy state, notably with the discovery of prognostic biomarkers defined as not easily visible regarding not yet triggered diseases.

To get a more global perspective, we must keep in mind that the overall body capacity evolves generally following a concave curve from birth to death through an increase up to an optimum and then a decline until the death (Figure 1). This overall line greatly varies between individuals likely due to genetic, epigenetic and environmental factors. Even if the 
optimum of maturity may then differ between the individuals, it appears, as we will see later with some specific physiological functions, that the higher the optimum, the later the organism dies or the later the disease occurs.

\section{Figure 1}

The classical system of concave curve for bodily skills

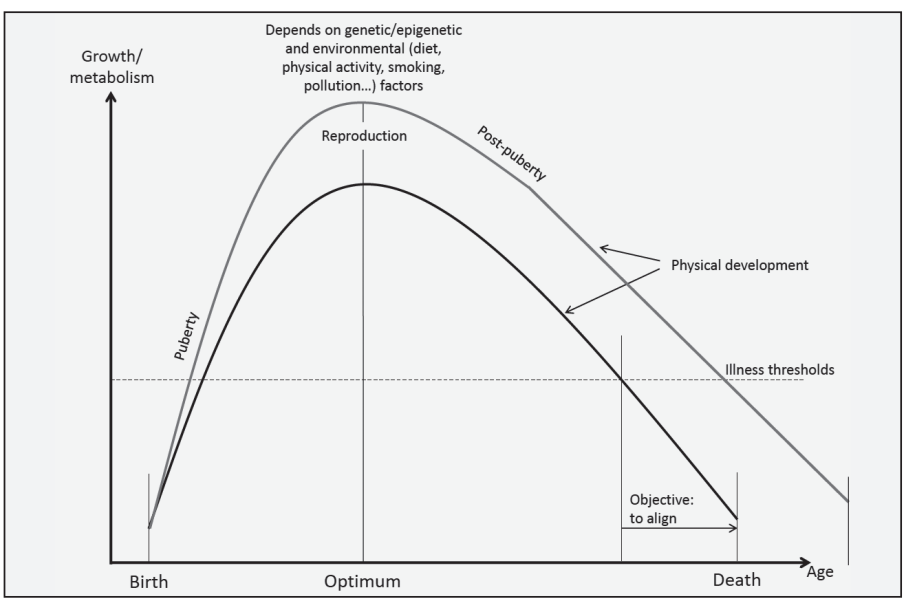

Based on this curve, the objective of preventive nutrition is then to bring closer the moment of disease occurrence with that of death, with the ultimate objective of making people dying in a healthy state.

At the level of human body there are 4 ubiquitous physiological systems that support the former view, and that are generally tightly interconnected: osseous, muscular, nervous and vascular systems. Other organs are not ubiquitously distributed within organism. In a healthy state at the first half of the life, each of these 4 systems evolved - in the second half of life - to attain a level of age-related chronic diseases including osteoporosis, sarcopenia, neuro-degenerative and cardiovascular diseases. Scientific evidence globally supports the fact that all the 4 functions described above follow the following scheme: growth, optimum and decline with age.

We then hypothesize that the objective of primary preventive nutrition should be to propose recommendations for reaching the highest possible optimum capacity, notably for the 4 physiological systems mentioned above. Then, whatever the decline of the system considered, it will not be likely to reach the threshold below which chronic disease occurs as osteoporosis, sarcopenia, cognitive decline and/or dementia, and cardiovascular diseases.

\section{The osseous system: evolution with age}

Evolution of bone density with age follows a concave curve as in Figure 1 with a phase of maturation, a peaks at about 30 years of age, then a decrease with risk of osteoporosis or osteopenia according to the initial optimum osseous capital (Figure 2) (14); note that bone loss in women occurs more rapidly than in men. We know today that it exist an osseous capital to reach and that the higher this capital the later the onset of osteoporosis. Therefore, an effective primary prevention will focus on building the higher optimum or peak of bone mineral density in order to delay the most the risk of osteoporosis and/or hip fracture, idealistically to prevent its occurrence until death.

\section{Figure 2}

Age-related changes in bone mass (from web site: http://cnx. org/content/col11496/1.6/, Jun 19, 2013. - OpenStax College)

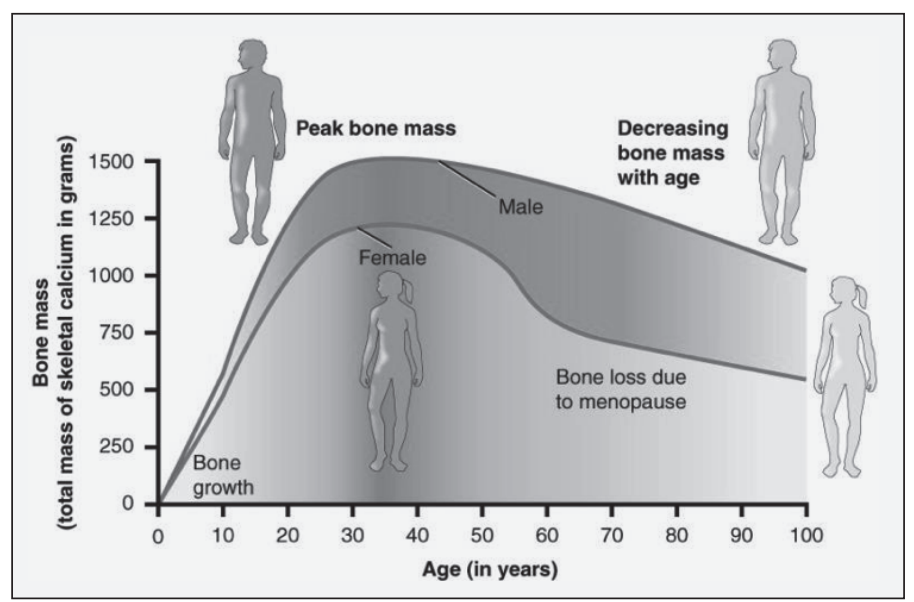

\section{The muscular system: evolution with age}

Interestingly, muscle mass and strength also follow a concave curve with age, i.e., with a growth phase, then a peak and finally a decrease (Figure 3) (15). It seems that, as for bone mass, muscle mass reaches a peak at around 30 years of age (16). Generally, sarcopenia, the loss of muscular mass (or around 3-5\% mass each 10 years) occurs from the age of 30 in men and at the entry to menopause in women. The resulting strength loss is accelerating beyond 50 years with a rate of about $1-2 \%$ per year.

\section{Figure 3}

A lifecourse model of sarcopenia (from Sayer et al. with permission of Springer (15))

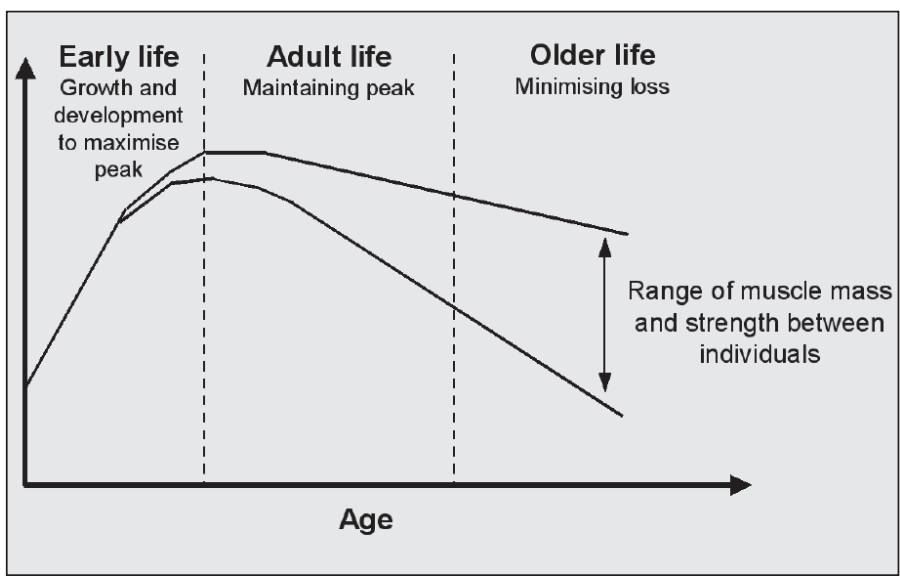


As for osteoporosis, an effective primary prevention will search to reach an optimum muscle mass peak at age of 30 . Concerning nutrition, it is therefore particularly important to identify factors that will help between 0-30 years of age to maximize muscle mass, be energetic input (diets) or output (physical exercise).

\section{The nervous system: evolution with age}

Data showing a concave curve linking cognitive capital or capacities with age during the overall life have not yet been published. However, it seems that, as for bone and muscle mass, it exists an optimum of mental health before cognitive decline. Thus, some cognitive abilities including word fluency, number, inductive reasoning, spatial orientation and verbal meaning, evolve in a manner similar to that described for bone and muscle mass evolution between 25 and 88 years of age (Figure 4) (17). Contrary to bone and muscle mass, the optimum seems to appear later around the age of 45 years (18). Schaie wrote in 1994: "The longitudinal gradients show at least modest gain for all abilities from young adulthood to early middle age. But there remain differences among abilities with respect to the attainment of peak age as well as the degree to which age changes accelerate with advancing age" (17). Again, we guess that the highest the cognitive functions at the optimum, the less the risk to have severe cognitive decline when ageing, and subsequent mental diseases such as all-type dementia or Alzheimer's disease.

\section{Figure 4}

Longitudinal estimates of mean $\mathrm{T}$ scores for single markers of the primary mental abilities (from 7-year within-subject data) (From Shaie (17) with permission of The American Psychological Association)

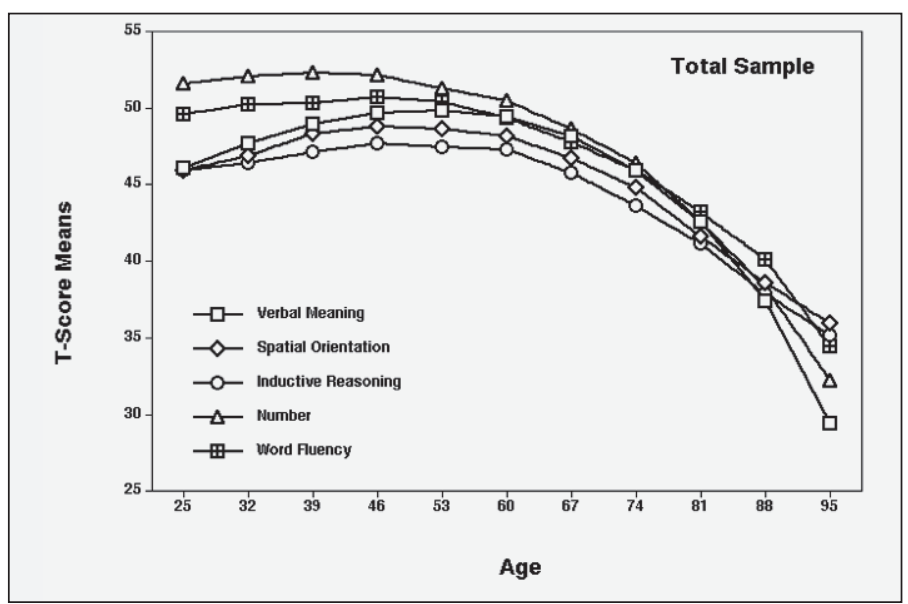

\section{The cardiovascular system: evolution with age}

Curves for the evolution of the vascular function from birth to death have not been yet published. However, some collected data may help having an idea of that evolution. For example, in the study by Lloyd-Jones et al., the percentage of normal, pre-hypertensive and hypertensive subjects (Framingham Heart Study cohort) changed in time (19). While normal subjects before the age of 60 were respectively $29.1 \%$ and $48.5 \%$ for men and women, these percentages decreased down to $11.7 \%$ and $15.0 \%$ at $60-79$ years old, and down to $8.2 \%$ and $6.3 \%$ at age $\geq 80$. Meanwhile, the number of pre-hypertensive and hypertensive subjects importantly increased with ageing, indicating a serious degradation of vascular function with age, similar to what was observed with nervous, muscular and osseous systems. The effect of age on blood pressure has been quite a lot studied. Thus, in another study, Heller et al. showed that between age of 40 and 59 years old, systolic and diastolic blood pressure increased by $2-7 \%(20)$. Such results were confirmed 4 years later by Brennan et al. in both men and women between 35 and 64 years old (21). These studies clearly suggest a degradation of vascular function with age, possibly from 35-40 years of age. Finally, over the period 20-85 years of age, Pannarale et al. also observed a regular increase in diastolic blood pressure (22). Concerning other risks of cardiovascular diseases, LDL cholesterol was observed to regularly increase between 20 and over 70 years old in both men and women (23). More globally, studying twelve cardiovascular diseases and association with lifetime risks, healthy life-years lost, and age-specific associations in 1,25 million people, Rapsomanik et al. recently observed a progressive and regular increase of lifetime risk in normal blood pressure and hypertension subjects from index age 30 years (Figure 5) (24).

\section{Figure 5}

Lifetime risk (95\% CI) of 12 different cardiovascular diseases in people with hypertension or normal BP from index age 30 years (hypertension was defined as systolic $\mathrm{BP} \geq 140 \mathrm{~mm} \mathrm{Hg}$ or diastolic $\mathrm{BP} \geq 90 \mathrm{~mm} \mathrm{Hg}$ or use of BP-lowering treatments or physician-recorded diagnosis at baseline). $\mathrm{BP}=$ blood pressure.

(from Rapsomaniki et al. with permission of Elsevier (24))

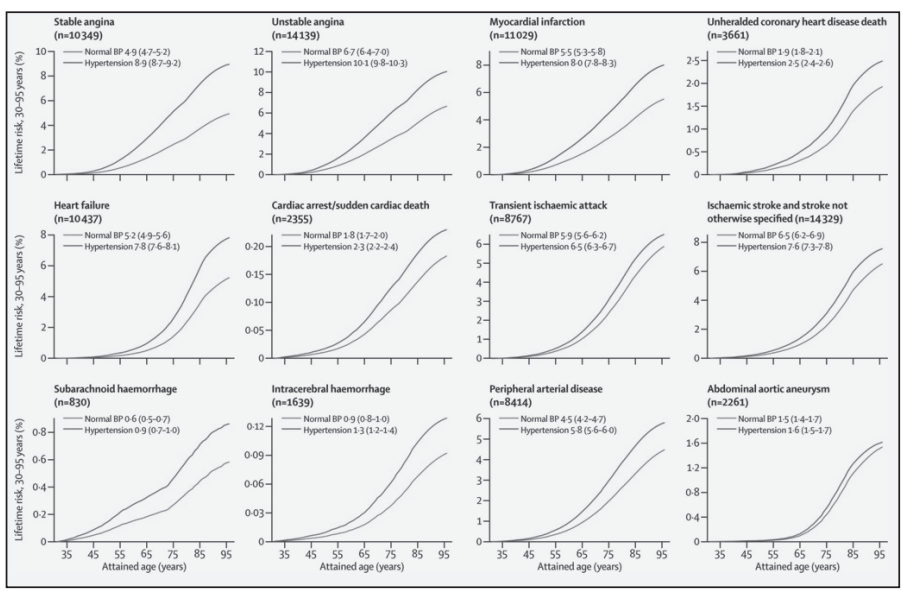

Otherwise, in another study, Black et al. found that maintaining a high level of fitness, or undertaking exercise 


\section{JNHA: NUTRITION}

training, prevents the age-related decline in the brachial artery vasodilator function evident in women, and that younger women demonstrated a greater brachial artery FMD than older sedentary women $(\mathrm{P}<0.05)(25)$. Even if these results were not observed in men, they showed that not only input (diet) but also output (physical exercise) may correct - via prevention cardiovascular functions and restore them to their healthy level.

Therefore, based on scientific evidence, it still remains difficult to definitively conclude that vascular system functioning also follows a concave curve from birth to death, similar to those observed for cognitive functions, bone density and muscle mass.

\section{The concept of compensation as regards to status}

Therefore, the neuro-vasculo-sarco-osteoporotic system should be at the optimum - as defined previously - for all of these components to be fully efficient to maintain the healthy state of the body. Since all these 4 systems are interconnected, from a holistic perspective, a weakness of one of them may lead to weaken the others leading to a phenotypic physiopathological state.

Therefore a new concept can be proposed, that of supportive compensation of one system by others to remain in a balanced healthy state. Indeed, to balance the whole body physiology (notably for curative nutrition in tertiary prevention), all functions may not re-equilibrate in the same way. These are all the paradoxes of an exclusively tertiary prevention too much based on a curative perspective focusing on only one of these systems. This again reflects a reductionist approach considering one organ or physiological system without taking into account the others in a more holistic approach (1).

For example, the will to prevent from muscle losses in old age via medicine or curative nutrition may be at first view positive; but, the osseous system must be reinforced in the same time to support the new excess muscle mass; and in the end the aging heart should be reinforced to supply blood to more bones and muscles to compensate their physiological needs. Similarly, it is proposed to improve protein status in older people with more protein supply, but are the aging kidneys able to support this without problem of insufficiency?

\section{The nodal regulator organs and the endocrine system}

A question remains unanswered: what is about other organs within human organism, e.g., heart, liver, kidney, or pancreas? They can be considered to act as homeostatic and/or buffer organs of the overall human organism and of its normal metabolism (or $\mathrm{HCM}$ ) in response to external stress (e.g., diets, physical exercise, temperature, microorganisms...) by specific functions.

Very schematically, heart regulates blood pressure, liver detoxifies organism from harmful xenobiotics and is a stock of amino-acids, kidneys are a kind of valve or sas that more or less opens according to the amount of waste, pancreas control glycemic homeostasis, gut sorts out what is consumed
- e.g., separating fiber fraction from nutrients -, lungs are what regulates the main input for human organism, that is oxygen, at the basis of all metabolic reactions. They are as dams or locks that regulate inputs and outputs toward the neuro-vasculo-sarco-osteoporotic system. With this perspective in mind, the neuro-vasculo-sarco-osteoporotic system appears as a sophisticated network linking all other organs. However, if any of these organs is deficient, the whole body suffers.

Finally, in addition to the neuro-vasculo-sarco-osteoporotic system and regulator organs, the whole is also regulated by the endocrine system that basically helps control organism homeostasis. Obviously, the endocrine system will therefore participate in the regulation of the HCM, which we will now tentatively describe.

\section{A new hypothesis: The concept of the Healthy Core Metabolism}

\section{Definition}

Beyond the neuro-vasculo-sarco-osteoporotic system, we hypothesize that it exists a basic metabolism that reflects the metabolic aspects of all healthy organs and remains invariant whatever the external stress, notably as regards with inputs (diet) and output (physical activity), but also as regards with external physical and psychological stress. This basic metabolism and the associated metabolites determined in healthy individuals is defined as the Healthy Core Metabolism (HCM). This HCM would be common and ubiquitous within human organism and would follow, as for bone and muscle mass, a concave curve. From a chemical aspect, this HCM would be a set of invariant metabolites whatever the external causes of stress, be $\operatorname{diet}(\mathrm{s})$, temperature or psychological stress, and thoroughly controlled by both the variation of the other metabolites generated by the stress conditions and the endocrine system. And when the organism goes from physiological to physiopathological and to pathological state, then the core metabolism will vary and deviate from its trajectory, reflecting deregulated metabolisms leading to a new unhealthy core metabolism distinct from the HCM. These different steps of the whole body system has been illustrated by the well-known diagram published by van der Greef et al. in 2004 (Figure 6) and often re-used $(8,26)$.

Indeed, in static rather than dynamic metabolomic studies that investigated evolution of metabolome following external intervention (e.g., diet, food or bioactives), the interpretation is almost exclusively on metabolome differences between test and control groups of healthy subjects, but there is always an invariant set of metabolites, usually representing a majority of measured metabolites, rarely shown and discussed. Whether this invariant metabolome is at the core of a healthy state is yet to be experimentally demonstrated. Moreover, according to our hypothesis, HCM is included into this invariant metabolome and can be determined if measurements are dynamically made to exclude the environment-induced changes of some 
metabolites over time. In addition, the variant metabolome might also be part of normal metabolism as controlling the invariant metabolome defined as HCM. This hypothesis opens another one consisting on considering the discriminating metabolites between test and control groups, as necessary to maintain the invariant HCM.

\section{Figure 6}

The development of disease from healthy (homeostasis within black dotted lines) to sub-optimal health and eventually an overt disease state. Biomarker patterns (for graphical reasons represented as a single line) are essential to describe the changes from normality to dysfunction (from ven der Greef et al. with permission of Elsevier (55))

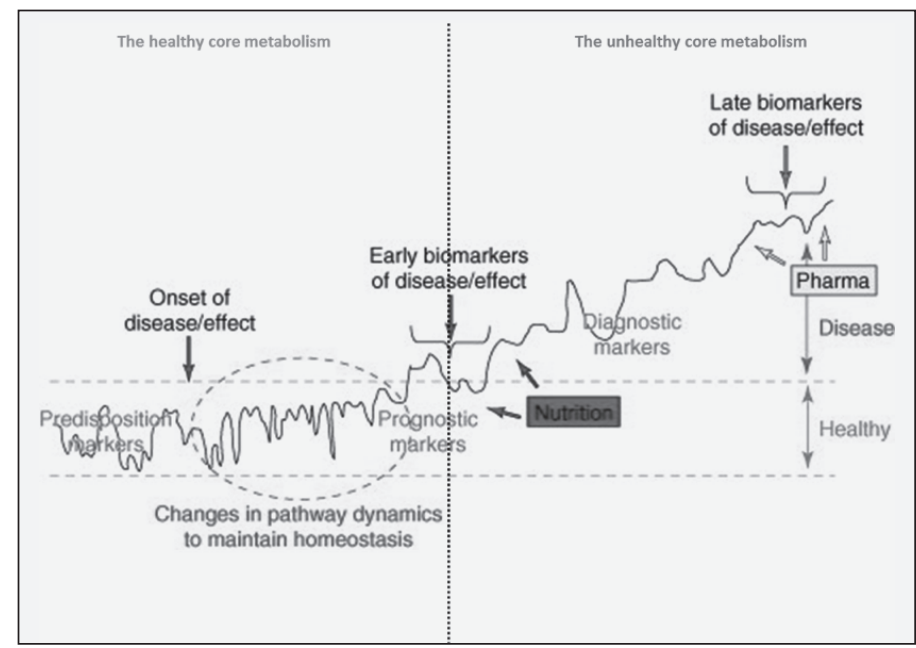

However, although being composed of a set of invariant metabolites, it is very likely that the HCM also evolves upon lifetime of healthy subjects with an inherent variation (i.e., its range) and optimum level depending on (epi-)genetic background and/or ethnicity, until reaching a variation that will lead to unhealthy core metabolism (Figure 7). The concept of natural metabolic variations has been highlighted in several inbred rat strains used as healthy controls: authors showed that homeostasis and "physiological plasticity can be achieved despite widespread divergences in glucose, lipid, amino acid, and energy metabolism in the host" (page 1675) (27).

\section{Natural factors of variation of the Healthy Core Metabolism}

Because of expected variation among individuals in endogenous metabolome, one of the main issues remains to characterize the HCM, its evolution upon time, its natural variation or range according to individuals (Figure 7).

From existing metabolomic longitudinal studies, several data can be collected that can help better apprehend what could be the HCM. Townsend et al. showed from 60 duplicate plasma samples donated by participants in the Nurses' Health Study and Health Professionals Follow-up Study that "CVs (coefficient of variation) were $<20 \%$ for $92 \%$ of metabolites and generally were similar by plasma anticoagulant type (heparin or EDTA) and fasting time" and that "ninety percent of metabolites were reproducible over 1-2 years within individuals" (page 1657) (28). It also seems that metabolites from healthy human plasma exhibit significant 24-h variations (29). For example, these authors showed that there is temporal profile of palmitoylcarnitine in human plasma and that among a total of 1069 metabolites that were detected, 203 (19\%) showed significant time-of-day variation (29). In another study, diurnal variations in both the urinary and salivary metabolic profiles were detected among subjects with BMI between 18.5 and 30 (30). In addition, in a review aiming at characterizing the normal degree of physiological variation in the absence of stimuli, it was concluded that inter-animal and diurnal variation, gender, age, diet, species, strain, hormonal status and stress have effects on the biochemical composition of urine of experimental animals, and that these are normal physiological variation (31). But, again, we think that such inherent variations help maintain the HCM.

\section{Figure 7}

A schematic representation of the evolution of the Healthy Core Metabolism upon lifetime. The Healthy Core Metabolism

is not constant at a defined time point but oscillate within a specific range depending on genetic background. Oscillation may changes upon external stresses to help the whole organism to regulate and remain stable. The highest the optimum of the Healthy Core Metabolism, the longer it will take time to decline upon ageing and the less it will be deregulated leading to a state of disease

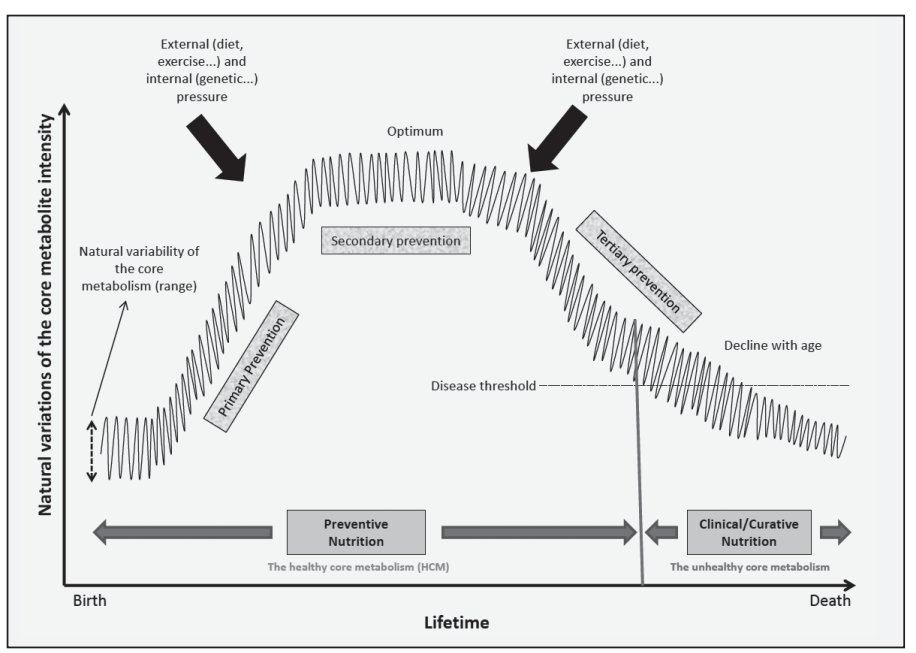

Genetic background is likely the most important factor of variation (27), and as wrote Trujillo et al.: "Although we should never lose the public health messages for health promotion, it is clear that not all individuals respond identically to a diet or to dietary components" (page 411) (32). For example, Rudkowska et al. showed in apparently healthy subjects differences in metabolic responses to an n-3 polyunsaturated fatty acids (PUFAs) supplementation depending the subjects 


\section{JNHA: NUTRITION}

were responders or non-responders (33). Further, it also exists cross-population differences in urinary metabolites that could be related to mainly genetic, but also dietary, and gut microbial factors (34).

Besides, as expected, there are also metabolomics differences according to the diet consumed. There are numerous studies in humans dealing with the variations of serum, plasma and urinary metabolites related to different diets, i.e., nutrimetabonomics (35). And most of them were dedicated to identify new biomarkers of food intake via the analysis of the now so-called food metabolome (36). In our laboratory, we notably shown that urinary metabolome of rats almost returned to its initial status upon around 48 hours when changing diet from whole-grain to refined wheat flour and conversely (37). Our hypothesis is that metabolic changes induced by the wholegrain diet diet helps to maintain the HCM as invariant as possible.

Another factor of variation that will affect the HCM is the disease state. Again, there are numerous metabolomics studies about this issue that showed distinct metabolic profiles between normal/healthy and test/ill subjects, with the aim to discover prognostic biomarkers (not easily visible but that appear just before the disease or metabolic deregulation is triggered) $(38,39)$. It notably concerns pre-diabetes $(40)$, cancer (41), nonalcoholic fatty liver (42), obesity (43), insulin resistance (44), and cardiovascular diseases (45). However, in this case, the HCM will progressively shift to an unhealthy core metabolism

Ageing is also an important factor of variation that can alter the HCM. However, for this factor, most studies were carried out in animal models such as rats (46) because it is not an easy task to follow a population or a group upon lifetime. In the study by Williams et al., urine metabolomes of rats were followed up to 20 weeks of age: while some metabolites increase with age (creatinine, taurine, hippurate and some amino acids/fatty acids), other decline (citrate and glucose/ myo-inositol), probably indicating that the HCM well evolved in time upon ageing (46).

Finally, effect of gender (31), exercise (47) and smoking (external stress) (48) must not be forgotten as well.

\section{How to experimentally approach, characterize and apply the healthy core metabolism?}

Today, the tools for studying the HCM exist. Obviously, there are all the 'omics' high throughput techniques, notably metabolomics to characterize the metabolome in biofluids such as urine, plasma, saliva, liver extracts, etc. Indeed, before going further, it is necessary to first search for this putative HCM, in other words to demonstrate whether or not it can be isolated and characterized.
Experimental characterization of the Healthy Core Metabolism: research design

As we previously discussed, the HCM is first what would be common to living beings whatever the external factors of variation, not what differentiate them. However, it may differ according to animal species.

A simple study to carry out would be first to evaluate whether in different metabolomics studies (initially aimed at identifying biomarkers of illness) the comparison of controls between different studies could reveal common metabolic signatures, i.e., the HCM. However, as these measurements were usually made as static ones, then a procedure has to be set to find out the HCM within the invariant metabolites.

Other quite simple experiments could be carried out to search for this HCM. Fundamentally, the objective is to define the HCM through the metabolites composing it and maintained at a level characterizing the «healthy state». Because many factors can modulate it (see above), fully genetic controlled living organism under confined physical environment can be used. Controlled healthy murine models fit well with those criteria. Easily harvested samples such as blood or urine can be daily collected from each individual for metabolomic experiments, for a period to be defined. These data will be first explored to define all the metabolites discriminating the samples obtained from one day to another and/or from one individual to another. Non discriminating metabolites thus obtained can then be explored as the one that are constitutive of HCM in those healthy individuals. At this stage, it is not necessary to identify the metabolites analyzed from their exact masses obtained with mass spectrometry. To check the core metabolism changes from healthy to a less healthy state, mild changes can be done by changing physiological (age) or environmental (fatty diet) parameters in a parallel experiment with the individuals from the same litter. By comparing the core metabolism obtained in each model, and before and after significant physiological changes occurred in the second experimental model, one can define the extent of changes in previously defined HCM. Obviously, many other interventions can be applied instead of only dietary changes and including physical exercise and drugs to better define the genuine plasticity of the body system to cope with these changes.

The practical aspects of the healthy core metabolism: how can we apply or practice this concept in real world settings?

The HCM should be a strong paradigm for improving preventive nutritional recommendations from birth to mid-life in order to reach the highest healthy metabolic peak at mid-life, then to maintain it as long as possible before natural decline (Figure 7). The underlying objective is obviously to limit the number of subject who will enter a disease state through significant decrease of diet-related chronic disease prevalence.

More practically, as nutrition researchers, our objective is to identify factors or ways of living (including diet and exercise) that will preserve the HCM. Therefore the HCM, a sum of 


\section{HEALTHY CORE METABOLISM AND PREVENTIVE NUTRITION}

metabolites found unchanged during a number of years to be determined, should be the central interest for the future clinical studies undertaken with methodologies presently developed on healthy volunteers. Basic science is also needed to determine how the changes of metabolites (newly synthesized and/ or the extent of their level changes) observed under given experimental conditions allow a maintenance of the HCM.

These reflections naturally lead us towards personalized nutrition $(49,50)$. For example, Chen et al. presented an integrative personal omics profile (iPOP) - an analysis that combines genomic, transcriptomic, proteomic, metabolomic, and autoantibody profiles - from a single individual over a 14 month period (39). The iPOP analysis uncovered extensive, dynamic changes in diverse molecular components and biological pathways across healthy conditions.

Basically, considering that everyone share a common HCM, an optimal personalized nutrition would be therefore a set of nutritional recommendations that would help to maintain as stable as possible the HCM of the subject (as that described above in the study by Chen et al.).

\section{A new preventive nutrition}

As stated by Jones et al. in 2012, "Advances that enable use of nutritional metabolomics to support complex biosystem models include the delineation of a conceptual grid for the exposome, a transition from disease-oriented to health-oriented nutrition, and a transition from a reductionist approach to one that incorporates non reductionist complex biosystems approaches" (page 197) (51). In other words, one urgently needs to move from a reductionnist and pharmacological to a holistic and preventive nutrition (1).

Primary preventive nutrition concerns healthy people as soon as birth to allow them living a long and healthy life. Referred to Figure 1, it is proposed that primary prevention is concerned by the ascendant curve during childhood and adolescence, while secondary prevention is concerned by the optimum reached during maturity or adulthood, and the focus on descendent curve during ageing as tertiary prevention. In this new perspective, primary preventive nutrition will consist in nutritional education and recommendations to reach the highest optimum possible as described in Figure 1. Then considering the natural decrease of the curve upon ageing, the disease threshold may either never be reached or very lately in age. In secondary preventive nutrition, the objective would be to maintain the optimum as long as possible. And finally the tertiary preventive nutrition would aim at influencing and delaying the descendent curve, an approach presently used with nutraceuticals targeting the (frail) elderly.

Besides primary, secondary and tertiary prevention, there is another concept of passive and active prevention. In each phase, we can then include a prevention taking into account or not the energy supply and expenses. In the case where we search for energetic equilibrium, one can speak of active prevention; the passive prevention being more to equilibrate foods through nutrients (amino-acids, omega-3 fatty acids...) or micronutrients (polyphenols, vitamins...) to optimize a determined or specific function, but without being worried by the general metabolic state or the HCM of the subject or a population. Another aspect of the active prevention can be also an involvement of the consumer in his (her) dietary regimen (purchase and culinary transformation of raw foods), whereas passive prevention might be a choice proposed by agro-food industry.

Today, numerous - but somewhat limited biological analyses - are proposed for the health assessment (cholesterol, LDLc, HDLc, $\mathrm{Na}, \mathrm{K}, \mathrm{Fe}, \mathrm{ASAT}$, ALAT, creatinine...). One future relevant question would be: is that assessment the same biological meaning than the HCM? For example, when the checkup indicates an increase in LDLc level, drug intervenes with an inhibitor of cholesterol synthesis. A thorough knowledge of the HCM might differentiate this curative action: in some cases, as regards with variations of other metabolites, cholesterol variation would be linked to an effect of adaptation and therefore useful for the system.

Finally, the concept of HCM that we developed in this paper is intended to be a tool for a better preventive nutrition, notably primary prevention. However, we are aware the HCM is probably not the only new preventive approach to holistic health, but it may help to lay new bases for new researches in the field of human nutrition.

We would like to conclude by the words of several researchers that support the usefulness of focusing on a primary preventive human nutrition in healthy subjects: "The field of metabolomics seeks to measure and understand the variation in metabolites in organisms, including humans. Metabolomics as an assessment strategy is designed to estimate the real-time realization of an individual's health potential. If we understand the variation in metabolism and how such variation relates to health, we can apply diet strategies to control metabolism" (page 1430) (50); "In order to develop insights into the health impact of certain food and food-components, we need to realize that health is adaptation set within a homeostatic range" (page 1074) (52); "Food constituents can be used to reset the health state, i.e. increase the ability to adapt" (page 299) (53); "Drugs are intended for, and evaluated in, sick people. Nutrients, and certainly nutrient intake recommendations, are first of all for well people" (page 1592) (54).

Disclosure statements: This research received no specific grant from any funding agency, commercial or not-for-profit sectors.

Conflict of interest: none

Authorship: Anthony FARDET and Edmond ROCK equally contributed to this work in formulating new hypotheses and paradigms, and in writing the manuscript.

Ethical Standards: None 


\section{JNHA: NUTRITION}

\section{References}

1. Fardet A, Rock E. Toward a new philosophy of preventive nutrition: from a reductionist to a holistic paradigm to improve nutritional recommendations. Adv Nutr 2014; 5: 430-446.

2. Popkin BM, Adair LS, Ng SW. Global nutrition transition and the pandemic of obesity in developing countries. Nutr Rev 2012; 70: 3-21.

3. Fries JF, Bruce B, Chakravarty E. Compression of morbidity 1980-2011: a focused review of paradigms and progress. J Aging Res 2011; 2011: 261702.

4. Ford ES, Bergmann MM, Kroger J et al. Healthy living is the best revenge: findings from the european prospective investigation into cancer and Nutrition-Potsdam Study. Arch Int Med 2009; 169: 1355-1362.

5. Fardet ARock E. The search for a new paradigm to study micronutrient and phytochemical bioavailability: from reductionism to holism. Med Hypotheses 2014; 82: $181-186$

6. World Health Organization W (1948) Preamble to the Constitution of the World Health Organization (signed on 22 July 1946 by the representatives of 61 States). In International Health Conference 19 June - 22 July 1946, vol. Official Records of the World Health Organization, pp. 100. New York

7. Patti GJ, Yanes O, Siuzdak G. Innovation: Metabolomics: the apogee of the omics trilogy. Nat Rev Mol Cell Biol 2012; 13: 263-269.

8. Fardet A, Boirie Y. Associations between diet-related diseases and impaired physiological mechanisms: a holistic approach based on meta-analyses to identify targets for preventive nutrition. Nutr Rev 2013; 71: 643-656.

9. Oberbach A, Blüher $\mathrm{M}$, Wirth $\mathrm{H}$ et al. Combined proteomic and metabolomic profiling of serum reveals association of the complement system with obesity and identifies novel markers of body fat mass changes. J Proteome Res 2011; 10: 47694788.

10. Floegel A, Stefan $\mathrm{N}, \mathrm{Yu} \mathrm{ZH}$ et al. Identification of serum metabolites associated with risk of type 2 diabetes using a targeted metabolomic approach. Diabetes 2013; 62: 639-648.

11. Duengen HD, Trippel TD, Tahirovic E et al. Validation of a metabolomics-based biomarker for asymptomatic, early stage heart failure. European J Heart Fail 2014; 16: 77-78.

12. Ni Y, Xie GX, Jia W. Metabonomics of human colorectal cancer: new approaches for early diagnosis and biomarker discovery. J Proteome Res 2014; 13: 3857-3870.

13. Rezzi S, Martin FPJ, Kochhar S. Defining personal nutrition and metabolic health through metabonomics. Oncogenes Meet Metabolism: From Deregulated Genes to a Broader Understanding of Tumour Physiology (Symposium Proceedings 074) 2008 4: 251-264.

14. Fordham JN (2003) Osteoporosis: Your Questions Answered, Your Questions Answered: Churchill Livingstone.

15. Sayer AA, Syddall H, Martin $\mathrm{H}$ et al. The developmental origins of sarcopenia. J Nutr Health Aging 2008; 12: 427-432.

16. Luna-Heredia E, Martin-Pena G, Ruiz-Galiana J. Handgrip dynamometry in healthy adults. Clin Nutr 2005; $24: 250-258$.

17. Schaie KW. The course of adult intellectual-development. Am Psychol 1994; 49: 304-313.

18. Singh-Manoux A, Kivimaki M, Glymour MM et al. Timing of onset of cognitive decline: results from Whitehall II prospective cohort study. BMJ 2012; 344: d7622.

19. Lloyd-Jones DM, Evans JC, Levy D. Hypertension in adults across the age spectrum Current outcomes and control in the community. JAMA 2005; 294: 466-472.

20. Heller RF, Rose G, Pedoe HD et al. Blood pressure measurement in the United Kingdom Heart Disease Prevention Project. J Epidemiol Community Health 1978, 32: 235-238.

21. Brennan PJ, Greenberg G, Miall WE et al. Seasonal variation in arterial blood pressure. Br Med J 1982; 285: 919-923.

22. Pannarale $\mathrm{G}$, Bebb $\mathrm{G}$, Clark $\mathrm{S}$ et al. Bias and variability in blood pressure measurement with ambulatory recorders. Hypertension 1993; 22: 591-598.

23. Schaefer EJ, Lamon-Fava S, Cohn SD et al. . Effects of age, gender, and menopausal status on plasma low density lipoprotein cholesterol and apolipoprotein B levels in the Framingham Offspring Study. J Lipid Res 1994; 35: 779-792.

24. Rapsomaniki E, Timmis A, George J et al. Blood pressure and incidence of twelve cardiovascular diseases: lifetime risks, healthy life-years lost, and age-specific associations in 1.25 million people. Lancet 2014; 383: 1899-1911.

25. Black MA, Cable NT, Thijssen DHJ et al. Impact of age, sex, and exercise on brachial artery flow-mediated dilatation. Am J Physiol-Heart Circul Physiol 2009; 297: H1109-H1116.

26. Rezzi S, Ramadan Z, Fay LB et al. . Nutritional metabonomics: Applications and perspectives. J Proteome Res 2007; 6: 513-525

27. Pontoizeau $\mathrm{Cm}$, Fearnside JF, Navratil V et al. Broad-ranging natural metabotype variation drives physiological plasticity in healthy control inbred rat strains. J Proteome Res 2011; 10: 1675-1689.
28. Townsend MK, Clish CB, Kraft P et al. Reproducibility of metabolomic profiles among men and women in 2 large cohort studies. Clin Chem 2013; 59: 1657-1667.

29. Ang JE, Revell V, Mann A et al. Identification of human plasma metabolite exhibiting time-of-day variation using an untargeted liquid chromatography-mass spectrometry metabolomic approach. Chronobiol Int 2012; 29: 868-881.

30. Walsh MC, Brennan L, Malthouse JPG et al. Effect of acute dietary standardization on the urinary, plasma, and salivary metabolomic profiles of healthy humans. Am J Clin Nutr 2006; 84: 531-539.

31. Bollard ME, Stanley EG, Lindon JC et al. NMR-based metabonomic approaches for evaluating physiological influences on biofluid composition. NMR Biomed 2005; 18 : 143-162.

32. Trujillo E, Davis CMilner J. Nutrigenomics, proteomics, metabolomics, and the practice of dietetics. J Am Diet Assoc 2006; 106: 403-413.

33. Rudkowska I, Paradis AM, Thifault E et al. Differences in metabolomic and transcriptomic profiles between responders and non-responders to an n-3 polyunsaturated fatty acids (PUFAs) supplementation. Genes Nutr 2013; 8: 411-423.

34. Dumas ME, Maibaum EC, Teague $\mathrm{C}$ et al. Assessment of analytical reproducibility of H-1 NMR spectroscopy based metabonomics for large-scale epidemiological research: the INTERMAP study. Anal Chem 2006; 78: 2199-2208.

35. Floegel A, Ruesten Av, Drogan D et al. Variation of serum metabolites related to habitual diet: a targeted metabolomic approach in EPIC-Potsdam. Eur J Clin Nutr 2013; 67: 1100-1108.

36. Primrose S, Draper J, Elsom R et al. Metabolomics and human nutrition. Brit J Nut 2011; 105: 1277-1283.

37. Fardet $\mathrm{A}$, Canlet $\mathrm{C}$, Gottardi $\mathrm{G}$ et al. Whole grain and refined wheat flours show distinct metabolic profiles in rats as assessed by a $1 \mathrm{H}$ NMR-based metabonomic approach. J Nutr 2007; 137: 923-929.

38. Armitage EG, Ruperez FJ, Barbas C. Metabolomics of diet-related diseases using mass spectrometry. Trends Anal Chem 2013; 52: 61-73.

39. Chen R, Mias GI, Li-Pook-Than J et al. Personal omics profiling reveals dynamic molecular and medical phenotypes. Cell 2012; 148: 1293-1307.

40. Wang-Sattler R, Yu ZH, Herder $\mathrm{C}$ et al. Novel biomarkers for pre-diabetes identified by metabolomics. Mol Syst Biol 2012; 8 :

41. Ng DJY, Pasikanti KK, Chan ECY. Trend analysis of metabonomics and systematic review of metabonomics-derived cancer marker metabolites. Metabolomics 2011; 7 : $155-178$.

42. Kalhan SC, Guo LN, Edmison J et al. . Plasma metabolomic profile in nonalcoholic fatty liver disease. Metabolism 2011; 60: 404-413.

43. She PX, Van Horn C, Reid T et al. Obesity-related elevations in plasma leucine are associated with alterations in enzymes involved in branched-chain amino acid metabolism. Am J Physiol-Endocrinol Metab 2007; 293: E1552-E1563.

44. Tai ES, Tan MLS, Stevens RD et al. Insulin resistance is associated with a metabolic profile of altered protein metabolism in Chinese and Asian-Indian men. Diabetologia 2010; 53: 757-767.

45. Yap IKS, Brown IJ, Chan Q et al. Metabolome-wide association study identifies multiple biomarkers that discriminate North and South Chinese populations at Didng risks of cardiovascular disease: INTERMAP Study. J Proteome Res 2010; 9: 664754.

46. Williams RE, Lenz EM, Lowden JS et al. The metabonomics of aging and development in the rat: an investigation into the effect of age on the profile of endogenous metabolites in the urine of male rats using H-1 NMR and HPLC-TOF MS. Mol Biosyst 2005; 1: 166-175.

47. Netzer M, Weinberger K, Handler M et al. Profiling the human response to physical exercise: a computational strategy for the identification and kinetic analysis of metabolic biomarkers. J Clin Bioinform 2011; 1: 34

48. Xu T, Holzapfel C, Dong X et al. Effects of smoking and smoking cessation on human serum metabolite profile: results from the KORA cohort study. BMC Med 2013; 11:

49. Xie GX, Li X, Li HK et al. Toward Personalized nutrition: comprehensive phytoprofiling and metabotyping. J Proteome Res 2013; 12: 1547-1559.

50. German JB, Watkins SM, Fay LB. Metabolomics in practice: emerging knowledge to guide future dietetic advice toward individualized health. J Am Diet Assoc 2005; 105: $1425-1432$

51. Jones DP, Park Y, Ziegler TR. Nutritional metabolomics: progress in addressing complexity in diet and health. Annu Rev Nutr 2012; 32: 183-202.

52. Hanekamp JC, Bast A, Calabrese EJ. Nutrition and health - Transforming research traditions. Crit Rev Food Sci Nutr 2015; 55: 1074-1080.

53. Bast AHanekamp JC. Chemicals and health - Thought for food. Dose-Response 2013; 11: 295-300

54. Heaney RP. Nutrients, endpoints, and the problem of proof. J Nutr 2008; 138: 15911595.

55. van der Greef J, Stroobant P, Heijden Rvd. The role of analytical sciences in medical systems biology. Curr Op Chem Biol 2004; 8: 559-565. 